\title{
ACTIVE CONTROL OF VIBRANT ACTUATORS WITH ADAPTIVE ADJUSTMENT OF THE REFERENCE.
}

\author{
Bertran, E. and Montoro, G.
}

Department of Signal Theory and Communications, Universitat Politècnica de Catalunya

c/ Jordi Girona 1-3, 08034 Barcelona (SPAIN) T. +3493 4015938 F.+3493 4015910

E-mail: bertran@tsc.upc.es

\begin{abstract}
An improved adaptive filter for eliminating periodic vibrations of unknown frequency in rotary machinery is presented. The proposed canceller is based on a usual bank of digital adaptive notch filters, each filter tuned in the cancellation of one harmonic. The amplitude and phase of each harmonic is adaptively adjusted by an LMS-based algorithm. Moreover, the central frequency of each notch filter is also adaptively adjusted (fine tuning). The resulting algorithm, tested in an industrial application, shows effectiveness in cancelling unknown periodic disturbances, reducing environmental noise and maintenance problems.
\end{abstract}

\section{INTRODUCTION}

Vibrations are usual in rotary machines, originating problems related to maintenance, components time of life and factory environmental noise. Active control is a powerful tool for reducing this problems, based on the generation of a signal that produces a vibration in the machine in the opposite sense of the undesired vibration. This process is similar to that of destructive interference [1]. Therefore the waveform of the vibration must be known in order to succeed with active compensators. The machine vibrations are composed of undesired subharmonics and harmonics of the rotor frequency. If vibrations are caused by rotary machinery with measurable speed it is feasible to keep track the frequency of rotation in order to identify the vibration wave [2].

The overall system is shown in the figure 1 , where the error signal $e(n)$ is the difference between the disturbance $d(n)$ and the canceller signal processed by the machine $G_{p}(z)[y(n)]$. The machine transfer function is $G_{p}(z)$. If disturbance and canceller signals are periodic waveforms they can be characterized by:

$$
\begin{gathered}
d(n)=\sum_{i=1}^{N} d_{i}(n)=\sum_{i=1}^{N} A_{i} \cos \left(\omega_{i} n\right)+B_{i} \sin \left(\omega_{i} n\right) \\
y(n)=\sum_{i=1}^{N} y_{i}(n)=\sum_{i=1}^{N} a_{i}(n) \cos \left(\omega_{i} n\right)+b_{i}(n) \sin \left(\omega_{i} n\right)
\end{gathered}
$$

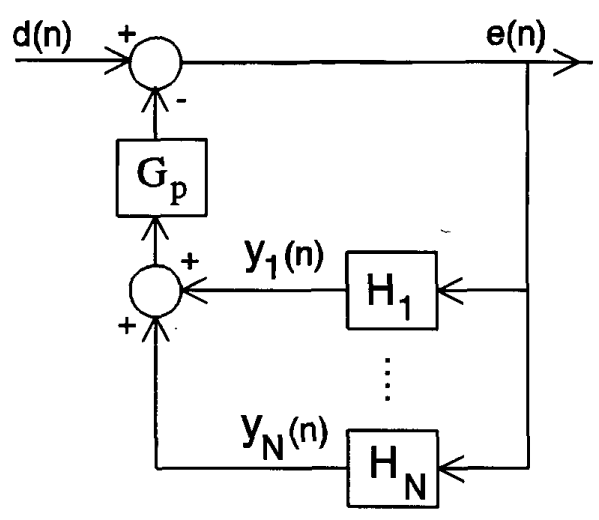

Fig. I Bank of notch filters.

\section{FEEDFORWARD CANCELLER}

The formulation of a second order discrete-time adaptive notch canceller with sinusoidal reference is:

$$
\begin{gathered}
y_{i}(n)=a_{i}(n) \cos \left(\omega_{i} n\right)+b_{i}(n) \sin \left(\omega_{i} n\right) \\
a_{i}(n+1)=a_{i}(n)+\mu_{i} e(n) \cos \left(\omega_{i} n\right) \\
b_{i}(n+1)=b_{i}(n)+\mu_{i} e(n) \sin \left(\omega_{i} n\right)
\end{gathered}
$$

the two adaptive weights being adjusted by means of an 
LMS algorithm. A detailed analysis of this sort of system is done in [1], where it is shown that using $Z$ transform a set of transfer-function relations are obtained. Using the common time-frequency mixed terminology, each notch filter has a bandpass transfer function $H_{i}$ as:

$$
\begin{gathered}
y_{i}(n)=H_{i}(z)[e(n)] \\
\text { being: } \quad H_{i}(z)=\mu_{i} \frac{z \cos \omega_{i}-1}{z^{2}-z 2 \cos \omega_{i}+1}
\end{gathered}
$$

For cancelling a periodic non-sinusoidal disturbance it is necessary a notch filter for each harmonic $\omega_{i}$, thus forming a bank of filters. If the frequencies to be cancelled are into the passband of the vibrating system then the system $G_{p}$ (machine transfer function) can be considered as a constant gain value $k_{p}$. Then, from figure 1 , the overall closed loop transfer function is:

$$
e(n)=\frac{1}{1+\sum_{i=1}^{N} k_{p} \mu_{i} \frac{z \cos \omega_{i}-1}{z^{2}-z 2 \cos \omega_{i}+1}}[d(n)]
$$

In order to have a stable narrow-band notch filter, the $\mu_{i}$ parameters must be sufficiently small. The adjustment of the parameter $\mu_{i}$ fixes the filter poles, thus providing the desired closed-loop frequency response. A small $\mu_{i}$ is related to a small bandwidth. In this case the canceller needs a more precise reference signal and the filter adjustment becomes critical. In rotary machinery the disturbance frequencies (and their harmonics) are usually estimated by measuring the rotor speed. Therefore, if there is an error in this measurement then the central frequency will not coincide with the frequency to be cancelled, and the vibration will be augmented.

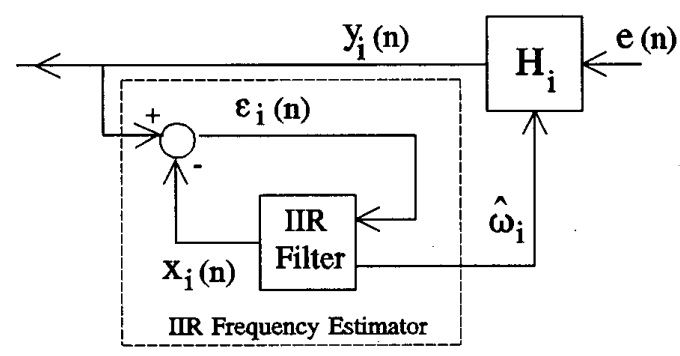

Fig.2 Frequency-tuned notch filter.

\section{ADAPTIVE ADJUSTMENT OF THE REFERENCE}

If an adaptive fine tuning of the central frequency is incorporated into a bank of notch filters then an algorithm able to cancel periodic disturbances of unknown frequency is obtained.

Each tuned notch filter can be represented as in figure 2. The unknown frequency to be cancelled is $\omega_{i}$ and initially the notch filter is centered at $\omega_{i}^{o}$ (an initial condition close to $\omega_{i}$ ). The narrow bandpass system $H_{i}$ (Eqn. 3) filters the error $e(n)$; therefore its output $y_{i}(n)$ is assumed to be a sinusoidal signal (the harmonic to be cancelled). In this case $y_{i}(n)$ can be considered as generated by the following discrete-time resonator [3]:

$$
y_{i}(n)=y_{i}(n-1) 2 \cos \omega_{i}-y_{i}(n-2)
$$

In order to estimate the frequency $\omega_{i}$ an adaptive filter is chosen as frequency estimator as in [4]:

$$
\begin{aligned}
x_{i}(n)= & x_{i}(n-1)\left(W_{i}(n)+2 \cos \omega_{i}^{o}\right)-x_{i}(n-2)+ \\
& +\left(\varepsilon_{i}(n-1) \cos \omega_{i}^{o}-\varepsilon_{i}(n-2)\right) \beta_{i}
\end{aligned}
$$

The constant parameters $\beta_{i}$ must be select according the behaviour of the frequency estimator. In the steadystate, this IIR adaptive system forces, if some conditions are accomplished, the estimation error $\varepsilon_{i}(n)$ to converge to zero. Hence, from Eqn. 6, a sinusoid is obtained with the same frequency than in Eqn. 5. The convergence depends on the correct selection of the variable parameters $W_{i}(n)$ and the constant $\beta_{i}$. From previous equations 5 and 6 , a new expression of the error is obtained:

$$
\begin{gathered}
\varepsilon_{i}(n)=y_{i}(n)-x_{i}(n)=F_{i}(z)\left[v_{i}(n)\right] \\
\text { where }: \\
v_{i}(n)=x_{i}(n-1)\left(2 \cos \omega_{i}-2 \cos \omega_{i}^{o}-W_{i}(n)\right) \\
F_{i}(z)=\frac{1}{1-z^{-1}\left(2 \cos \omega_{i}-\beta_{i} \cos \omega_{i}^{o}\right)+z^{-2}\left(1-\beta_{i}\right)}
\end{gathered}
$$

In [4] a similar frequency estimator is proposed, the $W_{i}(n)$ parameter being adjusted from an hyperstable algorithm. In the cases where $H(z)$ is unknown (totally or partially), it is not possible to assure the accomplishment of the SPR condition, and the IIR. filter design can lead to an unstable system.

We propose adjusting the adaptive parameter by using a gradient algorithm as follows: 


$$
\begin{aligned}
W_{i}(n+1) & =W_{i}(n)-\gamma_{i} \varepsilon_{i}(n) \frac{\partial \varepsilon_{i}(n)}{\partial W_{i}}=W_{i}(n)+ \\
& +\gamma_{i} \varepsilon_{i}(n) F_{i}(z)\left[x_{i}(n-1)\right]
\end{aligned}
$$

where $\gamma$ determines the convergence properties. If we approximate $\omega_{i} \simeq \omega_{i}^{o}\left(\omega_{i}\right.$ is unknown) then:

$$
F_{i}(z) \sim \hat{F}_{i}(z)=\frac{1}{1-z^{-1}\left(2-\beta_{i}\right) \cos \omega_{i}^{0}+z^{-2}\left(1-\beta_{i}\right)}
$$

the adaptive parameter adjustment and the estimated frequency being as follows:

$$
\begin{gathered}
W_{i}(n)=W_{i}(n-1)+\gamma_{i} \varepsilon_{i}(n) \hat{F}_{i}(z)\left[x_{i}(n-1)\right] \\
\hat{\omega}_{i}=\cos ^{-1}\left(\frac{W_{i}(n)}{2}+\cos \omega_{i}^{o}\right)
\end{gathered}
$$

\section{RESULTS AND CONCLUSIONS}

The canceller here presented has been implemented by using a PClabcard-812 included in a 486/DX2-66 PC. The sampling rate $f_{s}$ is 100 Samples/second. The vibrating system is a winding machine driven by a $\mathrm{DC}$ motor. A spectral analysis of the tachometer signal reveals the presence of three sub-harmonics whose relationship with the nominal speed $\left(f_{p}\right)$ are: $f_{p} / 30$, $\left(f_{p} / 30\right) \times 2$ and $\left(f_{p} / 30\right) \times 3$. The nominal speed $f_{p}$ is approximately $12.3 \mathrm{~Hz}$, so the frequencies to be cancelled are close to $0.41,0.82$ and $1.23 \mathrm{~Hz}$. The first harmonic $f_{p} / 30$ will be the frequency to be estimated. It has a value of $0.41 \mathrm{~Hz}$, corresponding to a digital frequency of 0.0257 . For the estimated frequency is considered an initial condition of 0.028 , obtained from a previous spectral study of the noisy system. The best cancellation results have been obtained by using the following set of parameters:

$$
\begin{gathered}
\gamma_{i}=4 \cdot 10^{-7} \quad \mu_{i}=-3 \cdot 10^{-3} \quad \beta_{i}=0.35 \\
i=1,2,3
\end{gathered}
$$

Several sampled waveforms are shown. In figure 3 it is shown the error $e(n)$ by sampling the tachometer signal. The cancellation done by a conventional adaptive notch filter is shown below in figure 3. In this case, the failure in the cancellation is due to the uncertainty in the frequency to be cancelled: a digital frequency of 0.028 is considered. Figure 4 shows the evolution of the estimated central frequency of the first notch filter, used in each iteration. The algorithm converges to a final value close to the correct frequency (0.0257). Moreover, from the comparison between figures 3 and
4 is reveled and improvement of the cancellation obtained with the proposed adaptive notch filter in front of a conventional structure.

\section{REFERENCES}

[1] Kuo, S.M. and D.R. Morgan, "Active Noise Control Systems" (John Wiley, 1996).

[2] Jiang, F., H. Tsuji, H. Ohmori and A. Sano, "Adaptation for Active Noise Control", IEEE Control Systems Magazine, pp. 36-47, December 1997.

[3] Bodson, M. and S.C. Douglas, "Adaptive Algorithms for the Rejection of Sinusoidal Disturbances with Unknown Frequency", Automatica, Vol. 33, pp. 2213-2221, December 1997.

[4] Padmanabhan, M. and K. Martin, "A Second-Order Hyperstable Adaptive Filter for Frequency Estimation", IEEE Trans. on Circuits and Systems II, Vol. 40, pp. 398-403, June 1993.

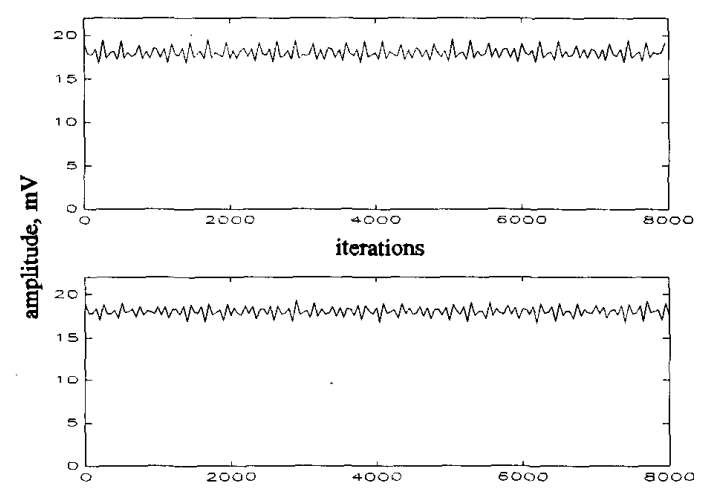

Fig.3 Tachometer signal e(n): without canceller (above) and with a conventional adaptive notch canceller (Eqn. 2) (below).
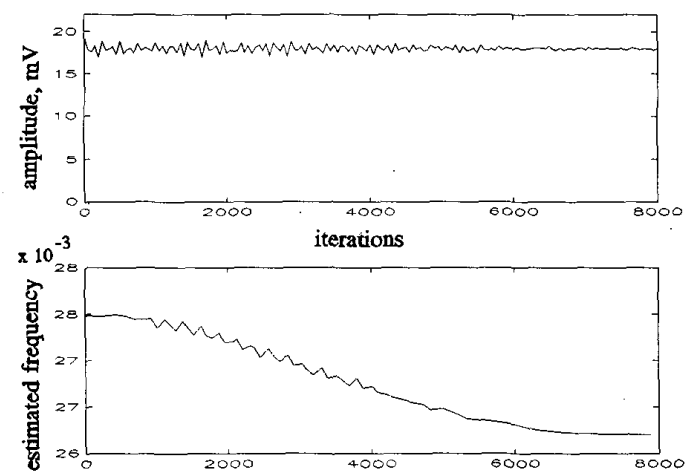

Fig. 4 Tachometer signal e(n) with the proposed frequency-tuned notch filters (above), and evolution of the estimated frequency $\hat{\omega}_{1}$ (below). 